Meta

Journal des tradlucteurs

Translators' Journal

\title{
Intraduisible ?... Pas français !
}

Serge Rajsfus-Raffet

Volume 21, numéro 1, mars 1976

La traduction et l'entreprise

URI : https://id.erudit.org/iderudit/003905ar

DOI : https://doi.org/10.7202/003905ar

Aller au sommaire du numéro

Éditeur(s)

Les Presses de l'Université de Montréal

ISSN

0026-0452 (imprimé)

1492-1421 (numérique)

Découvrir la revue

Citer cet article

Rajsfus-Raffet, S. (1976). Intraduisible ?... Pas français ! Meta, 21(1), 22-26.

https://doi.org/10.7202/003905ar d'utilisation que vous pouvez consulter en ligne.

https://apropos.erudit.org/fr/usagers/politique-dutilisation/ 


\title{
Intraduisible?... Pas français!
}

\begin{abstract}
Mini-mélodrame moderne et vrai se voulant édifiant et instructif et montrant comment fonctionne une entreprise importante et moderne de traduction en décrivant par le menu les événements qui s'y sont réellement déroulés un jour qui n'a de particulier que d'être le dernier de l'année dernière.
\end{abstract}

L'hiver, à huit heures trente du matin, à Paris, il fait encore nuit, même dans le seizième arrondissement. Surtout le jour de la Saint-Sylvestre. Et froid : non que la température soit tellement basse, mais il fait humide et les gens ne sont jamais très bien couverts. Les Parisiens, en fait, sont mal équipés en toutes saisons parce qu'il n'y a pas vraiment de saisons à Paris. Il y fait toujours mauvais.

C'est le facteur qui passe le premier au bureau, dépose un paquet de courrier et continue sa tournée. Il est antillais et paradoxalement semble le seul à n'avoir pas froid.

Albert et Jeanne arrivent souvent très tôt au bureau. Jeanne commence à ouvrir le courrier et le passe à Albert. Albert le trie. La moitié des commandes de la journée arrivent par le premier courrier du matin. Elles sont mises à part en un petit tas, chacune dans une chemise transparente verte, avec l'enveloppe dans laquelle elles sont arrivées. Car de temps en temps des clients oublient de mettre leur adresse à l'intérieur : il est commode, alors, de retrouver leur adresse et même leur nom imprimé sur l'enveloppe. Celle-ci porte également la date d'envoi : encore une information utile lorsque le client s'impatiente de ne pas recevoir son texte. Car il croit peut-être nous l'avoir envoyé la semaine précédente, mais sa secrétaire, pour des raisons qui nous échappent ainsi qu'à lui et peut-être même à elle, a attendu trois jours avant de le mettre à la poste, ou n'a pas mis suffisamment de timbres dessus. Ces détails qui semblent tellement loin de la traduction sont d'une importance majeure dans notre métier.

Pour les distinguer, les demandes de devis et d'informations sont mises dans des chemises transparentes rouges. Le rouge implique l'urgence et la réponse à ces demandes de renseignements de la part des clients doit intervenir dans la journée, au plus tard le lendemain.

Quelquefois, des clients retournent des documents traduits en faisant des observations sur la qualité des traductions. On a choisi pour ces envois de les mettre dans des chemises transparentes jaunes. Il doit bien y avoir une explication psychologique profonde à ce choix de couleur. La plupart du temps, ces renvois de traductions ont deux causes. Il y a l'entreprise dont un ingénieur a passé quelques mois en stage, il y a vingt ans, dans un pays de langue espagnole et qui ne retrouve pas dans notre traduction les quelques mots de patois ou de jargon qu'il avait 
retenus. Désemparé, mais voulant montrer sa science des langues, il va voir son directeur avec le texte et le critique. Le directeur, qui ne connaît pas l'espagnol, faisant confiance à ce vieil ingénieur si sérieux dans son travail, nous le renvoie. L'autre cas est celui de l'entreprise qui nous demande de traduire un document et envoie la traduction à sa succursale dans le pays où l'on pratique la langue en question, avec demande de commentaires. Que feriez-vous si vous receviez de l'autre bout du monde un document traduit dans votre propre langue et si on vous demandait de le critiquer? Sans doute le critiqueriez-vous. Il n'existe pas de traduction absolue et définitive et toute traduction peut toujours être relue, révisée, modifiée, corrigée, quelquefois même améliorée... Ce bureau lointain va donc corriger notre traduction, ne serait-ce que pour montrer qu'il a travaillé dessus et pour faire plaisir au siège social. Et d'ailleurs, il est intéressant, amusant, de corriger une traduction, de jouer les créateurs, de montrer et de se montrer sa science et sa compétence. Le texte va donc revenir à son expéditeur avec des corrections. Comme celui-ci ne peut pas juger de la justesse et de la nécessité de ces corrections, il va d'abord s'inquiéter, puis pester contre nous avant de nous renvoyer le texte que nous avions traduit. Ou tout simplement nous dire que notre traduction n'était pas bonne. Puisqu'elle est revenue avec des corrections, n'est-ce pas? Et pendant ce temps, il retient le paiement de notre facture, ce qui est toujours bon à prendre pour lui. Dans les deux cas, c'est Albert qui s'efforcera de calmer ces clients et de leur expliquer qu'en matière de traduction, deux opinions différentes peuvent être également justes. Dont la nôtre.

Dès qu'Étiennette, Catherine et Roland arrivent, ils saisissent le paquet de chemises vertes et rouges et en commencent le tri. Martine, la comptable, arrive en général un peu plus tard et trouve déjà les chèques, traites, factures de fournisseurs, relevés de banque et autres documents comptables sur son bureau. Antoinette l'aide à les traiter, cela leur prendra une bonne partie de la journée. Le reste du temps, elles préparent les déclarations, fiscales et autres, et rappellent aux clients en retard qu'il est temps de payer les factures trop anciennes.

Malcolm, Gilbert, Christian et Myriam sont déjà assis derrière leurs machines à écrire et terminent quelques traductions courtes arrivées la veille et qui doivent être livrées avant midi.

Les commandes continuent à arriver toute la journée. Par courrier - car il y a trois distributions ordinaires de courrier par jour à Paris, plus les distributions par porteur spécial pour le courrier exprès - par messager ou amenées directement par les clients ou leurs employés. Elles sont triées selon quatre critères : l'urgence, la longueur des documents à traduire, les domaines techniques et les langues. Les télex constituent une catégorie de commandes à part. Le bureau est équipé du télex et reçoit toute la journée des télex à traduire qui lui sont envoyés par les clients qui disposent eux-mêmes du télex. Ces télex doivent être traduits immédiatement. En général, il s'agit de télex rédigés en allemand, espagnol, italien, hollandais ou anglais à traduire en français ou inversement. C'est Jeanne qui les reçoit, les analyse, ouvre les dossiers-clients correspondants (dans des chemises vertes, bien sûr) et les fait traduire immédiatement, toutes affaires cessantes, par l'un des membres de l'équipe des traducteurs permanents. Elle traduit elle-même d'espagnol et d'anglais en français et peut aussi traduire les télex vers l'espagnol. 
Les commandes urgentes peuvent être courtes: articles de journaux, textes publicitaires, annonces qui doivent passer dans la prochaine édition de plusieurs quotidiens qui sont nos clients. Malcolm traduit vers l'anglais, Étiennette vers l'allemand, Gilbert, Christian et Myriam vers le français. Dans la plupart des cas, les documents sont traduits dans l'heure, au plus tard dans la journée et repartent chez les clients par télex, pneumatique ou messager. Nous avons pour cela deux messagers qui sillonnent Paris en moto d'un bout de l'année à l'autre et nous utilisons de plus un service de coursiers extérieur à notre entreprise.

Toutes les autres commandes sont traitées par des traducteurs dits \& indépendants $»$. Ce sont des traducteurs spécialisés dans des domaines très techniques, ayant une connaissance approfondie de ces domaines et qui de plus ont le goût de ce qu'ils font et aiment le faire à leur rythme. Ces traducteurs traduisent uniquement dans leur langue maternelle et seulement sur des documents traitant de sujets qu'ils connaissent parfaitement. Si la traduction n'est pas une création originale au même titre que la préparation d'un article ou d'un mémoire, elle n'en exige pas moins une réflexion, une concentration qui font mauvais ménage avec les impératifs d'une production programmée et accélérée. Selon la nature du texte à traduire aussi bien que selon sa forme physique et intellectuelle, un même traducteur pourra produire en un jour de 800 à 3000 mots de texte bien traduit. Si le document confié à un traducteur est relativement long et s'il est seul à travailler dessus, il est inutile qu'il perde chaque matin et chaque soir du temps pour venir au bureau. Il vaudra bien mieux qu'il travaille chez lui, tranquille, à son aise et à son rythme. Il travaillera tôt le matin s'il y est disposé, ou tard le soir si d'un coup l'inspiration lui vient. Cela ne serait pas possible s'il était astreint à un horaire fixe au bureau.

Lorsqu'un texte à traduire est très long - 100 pages ou plus - et si le délai imposé est trop court pour qu'un même traducteur puisse l'achever à temps, nous organisons une équipe de traducteurs compétents dans le domaine traité par le document et la langue de ce document et dont la langue maternelle est la langue dans laquelle il faut le traduire. Cette équipe, constituée de deux ou plusieurs traducteurs, comprend aussi une ou plusieurs dactylos et un coordinateur réviseur qui organise le travail des traducteurs, veille à l'homogénéité de la terminologie et révise les documents avant leur dactylographie définitive.

Ainsi, un grand nombre de traducteurs dits « indépendants » consacrent en pratique l'essentiel de leur temps et de leur activité à notre entreprise mais conservent une grande liberté et peuvent toujours refuser certains des travaux que nous souhaitons leur confier.

La matinée s'avance ainsi, ponctuée d'innombrables coups de téléphones (le bureau est équipé de sept lignes extérieures et douze postes intérieurs et cela est à peine suffisant) de clients qui demandent des renseignements, veulent savoir quand leurs traductions seront prêtes, changent d'avis et modifient leurs textes - ou même annulent la commande de traduction pour un document que nous avons déjà fini de traduire - ou de traducteurs qui voudraient bien travailler pour nous, ou de démarcheurs qui veulent vendre de l'espace publicitaire ou des fournitures de bureau. 
Tout au long de la journée, les traducteurs rapportent les travaux qu'ils ont exécutés. Ces travaux sont aussi quelquefois rapportés par les coursiers, afin d'économiser le temps précieux des traducteurs et de les laisser avancer plus vite ce qu'ils sont en train de faire. Lorsque les travaux sont peu urgents, ils sont acheminés par courrier dans les deux sens.

Dès qu'une traduction est terminée par le ou les traducteurs qui y ont été affectés, elle est revue, corrigée si nécessaire, dactylographiée à nouveau. Puis elle est analysée en vue de sa facturation tandis que sont calculés les honoraires des traducteurs et des autres personnes qui y ont travaillé. Elle est ensuite mise sous chemise spéciale avec un bon de livraison, une facture et des bons de commande pour les prochaines fois et enfin cxpédiée au client par messager ou courrier, quelquefois par fret aérien lorsqu'il s'agit de la faire parvenir très rapidement à un client domicilié à l'étranger. Ou mise en attente si c'est le client lui-même qui doit venir en prendre livraison.

La journée passe vite. À l'heure du déjeuner, le personnel se divise en deux afin qu'une permanence assure le service de façon continue. La nuit commence à tomber vers cinq heures. Les lumières s'allument mais la fièvre ne tombe pas. Car c'est vers la fin de l'après-midi que les clients s'aperçoivent de ce qu'ils ont oublié de nous demander de traduire le matin et les coups de téléphone deviennent plus frénétiques, plus pressants. La sarabande des motards casqués, dégoulinants de pluie, des enveloppes à la main, s'accélère. Babette, à la réception, toujours imperturbable, a le téléphone vissé à l'oreille et pianote de plus en plus vite sur les boutons de son standard pour passer les communications, tandis que les visiteurs se font plus nombreux.

Enfin, vers sept heures, il ne reste plus que trois personnes au bureau. Dehors, la nuit est noire. On dit que Paris est la ville-lumière. Peut-être intellectuellement. Car les rues sont souvent sombres, et je connais des capitales mieux éclairées dans le monde. Étiennette finit de classer quelques dossiers et prépare son travail pour le lendemain matin. Albert, embarrassé, explique à sa femme qu'il a bientôt fini sa journée et va rentrer à la maison et que les invités ont bien de la chance de sortir si tôt de leurs bureaux et qu'ils peuvent bien l'attendre en buvant quelque chose. Dans un coin Malcolm tape la traduction d'un document urgent que le client doit venir chercher demain matin à la première heure.

Demain, c'est le Nouvel An. La moitié des Parisiens sont en vacances, à la montagne ou au Club Méditerranée. L'autre moitié se prépare à faire la fête. Les boutiques restent ouvertes tard, pour satisfaire les clients attardés et dessinent deux fleuves de lumière qui se reflètent sur l'asphalte humide le long des rues des quartiers commerçants. Ce fut une belle année. On espère que la prochaine sera encore meilleure. Puis ce sera la suivante. Ainsi va la vie. Soudain, dans le silence feutré, le téléphone sonne. Cela surprend presque, le bureau commençait à s'enfoncer dans le silence. Dans le fond, l'aspirateur de la femme de ménage ronronne doucement. Un avocat nous appelle. Son télex est en train de recevoir un long message de Caracas en espagnol. Là-bas, ils commencent à peine l'après-midi. Par téléphone, on lui a annoncé que ce message télexé fera trois mille mots - un télex de quatre mètres de long. Son cabinet a besoin de la traduction pour le deux janvier au matin. 
Pouvons-nous les dépanner? Il propose d'apporter le télex lui-même dans une heure ...

La traduction sera faite. Mais les invités d'Albert et de sa femme mangeront un soufflé froid et plat et un rôti trop cuit. Étiennette qui était invitée en banlieue pour fêter le réveillon se perdra et arrivera chez ses amis après minuit, c'est-à-dire avec un an de retard et Christian, rattrappé de justesse au moment où il partait pour la gare, passera la première journée de l'année penché sur sa table de travail au lieu de faire du ski. Philosophe, il conclut que cela lui évitera peut-être de se casser une jambe.

La traduction, si on veut s'en occuper sérieusement, demande beaucoup d'abnégation.

Serge RaJsfus-Raffet 